\title{
Princípios de gravação magnética e registro de som em fios
}

Magnetic recording principles and sound record on wires

\author{
Nilson Evilásio de Souza Filho*1이, João Paulo Gazola², Artur Harres de Oliveira ${ }^{2}$, \\ Nelson Guilherme Castelli Astrath ${ }^{3}$ \\ ${ }^{1}$ Universidade Federal de Santa Maria, Engenharia Acústica, Rio Grande do Sul, RS, Brasil \\ ${ }^{2}$ Universidade Federal de Santa Maria, Departamento de Física, Rio Grande do Sul, RS, Brasil \\ ${ }^{3}$ Universidade Estadual de Maringá, Departamento de Física, Paraná, PR; Brasil
}

Recebido em 02 de Junho, 2018. Aceito em 03 de Setembro, 2018.

\begin{abstract}
Construímos uma plataforma de gravação magnética de fios a partir da sucata de um clássico gravador WebsterChicago. O gravador de fios recuperado utiliza um motor para controlar engrenagens acopladas e mover carreteis que transportam um fio que passa por uma cabeça indutora. O som captado por um transdutor pode ser gravado diretamente no fio pela cabeça indutora e a mesma cabeça faz a leitura por um processo recíproco. Comparamos o sinal gravado direto da cabeça de gravação com o mesmo sinal com correção de distorção (polarização de corrente contínua e corrente alternada) e discutimos a relação sinal/ruído e a distorção de gravações em fios de aço. Palavras-chave: Registro de som, gravação magnética; fio de aço, relação sinal ruído.
\end{abstract}

We built a magnetic wire recording platform from the scrap of a classic Webster-Chicago recorder. The restored wire recorder uses a motor to control coupled gears and to move trolleys carrying a wire passing through an inductive head. The sound picked up by a microphone is recorded directly within the wire by the inductive head and the same head performs the reading by a reciprocal process. We compared the recorded signal directly from the recording head with the same signal with distortion correction (DC and AC bias) and discussed the signal to noise ratio and distortion of recordings on steel wires.

Keywords: Sound record, magnetic recording, steel wire, signal noise relation.

\section{Introdução}

É possível classificar as tecnologias clássicas de registro fonográfico em: gravação mecânica, gravação magnética e gravação óptica. A evolução de cada técnica de registro está vinculada ao suporte físico de gravação (media) em que o som é registrado [1. No presente trabalho, discutiremos o primeiro gravador magnético e seu primeiro suporte de gravação, um material ferromagnético na forma de fio.

O conceito de registro e reprodução de som por meio magnético foi publicado pela primeira vez em 1888, por Oberlin Smith 2]. Seis anos mais tarde, em 1894, o engenheiro dinamarquês Valdemar Poulsen criou o gravador magnético conhecido como Telegraphon [3]. O principal componente deste tipo de gravador é a cabeça indutora, que tem a função de magnetizar um meio material com informações sonoras. O primeiro suporte de gravação magnético utilizado comercialmente foi o fio de aço. $\mathrm{O}$ gravador de fios veio para substituir os gravadores mecânicos supostamente ultrapassados.

No entanto, dentro do período de vinte anos, de 1900 a 1920, o gravador de fios entrou em uma fase inicial de

*Endereço de correspondência: nilson.evilasio@eac.ufsm.br obsolescência e foi superado pela própria máquina que deveria ser melhorada, o fonógrafo [1.4].

O gravador de fios voltou a ser utilizado durante a Segunda Guerra Mundial e então entrou em uma era de sucesso sem precedentes, assumindo a posição do gravador de som mais popular da época. A máquina de gravação de fio utilizada entre 1945 e 1952, era compacta e podia ser transportada facilmente. O sistema era destinado tanto para uso profissional quanto para uso doméstico. As vendas anuais do dispositivo fabricado pela Webster-Chicago, atingiram dezenas de milhares de unidades [4].

A gravação em fios magnéticos é uma técnica excessivamente sensível. O fio é fino e flexível, fácil de enrolar e manipular. Entretanto, ele possui limitações claras no que diz respeito à cortes, edições e espaço de armazenamento. No final da década de 1950, a gravação em fio tornou-se uma técnica obsoleta, sendo substituída pela gravação em fita magnética [5].

Apesar da rápida obsolescência dos fios como media, as teorias mais simples do magnetismo e os conceitos tecnológicos de gravação de fios formam a base para o entendimento de toda a evolução da gravação magnética. Indiscutivelmente, a gravação magnética e seus diferentes tipos de suportes, têm importância fundamental na 
evolução da indústria fonográfica, da eletrônica, da computação e armazenamento de dados. Esta nota de aula apresenta os princípios da gravação magnética e a problemática tecnológica de seu suporte de gravação original, a fim de ilustrar as causas da obsolescência rápida da primeira media magnética.

\section{Técnicas de Gravação}

\subsection{Gravação magnética longitudinal}

Para compreendermos o processo de gravação e leitura de informação em medias magnéticas, é fundamental entender o mecanismo físico por trás dele, ou seja, o comportamento dos materiais ferromagnéticos. Basicamente, ferromagnetismo é o mecanismo responsável por certos materiais formarem ímãs permanentes. Um pedaço de ferro, por exemplo, possui em seu interior uma série de momentos magnéticos distribuídos ao longo de seu volume. Cada um destes momentos magnéticos pode ser representado por um pequeno ímã.

Ao aplicarmos um campo magnético sobre um material ferromagnético, estes ímãs tenderão a se alinhar com o campo, modificando assim sua configuração magnética [6] . Podemos então atribuir ao material, ou a uma região deste material, um valor de magnetização, que é definida como o seu momento magnético por unidade de volume.

Uma das propriedades mais marcantes dos ferromagnetos é que eles podem manter este novo estado magnético, total ou parcialmente, mesmo quando o campo magnético externo é removido. Assim, é possível codificar informação na configuração magnética do material. Obviamente, para que o material seja uma media útil é preciso ler esta informação sem destruí-la. Isto é viável pois o estado dos momentos magnéticos pode gerar um campo magnético nas proximidades do material, que depende da configuração magnética específica. Este campo pode ser detectado e a informação lida.

Em um processo de gravação magnética, o campo magnético utilizado para orientar a magnetização de determinada região do material é produzido por uma corrente elétrica que percorre uma bobina toroidal (cabeça de indução). Tal corrente é gerada por um transdutor eletroacústico, que converte energia sonora em um sinal elétrico [7]. O conceito de gravação magnética longitudinal é utilizado tanto em fios quanto em fitas. A diferença entre eles, além da media, é que na gravação em fita, a cabeça indutora é uma superfície uniforme, enquanto que para o fio a cabeça deve ter uma fenda estreita.

A Figura 1 apresenta o sistema de gravação magnética descrito por Oberlin Smith; o suporte de gravação passa por uma cabeça magnética em ambos os processos: gravação e leitura. Durante o processo de gravação, o suporte de gravação passa com velocidade $v$ pelo campo gerado no gap da cabeça de gravação, e a magnetização do material fica alinhada de acordo com a direção instantânea e magnitude do campo.

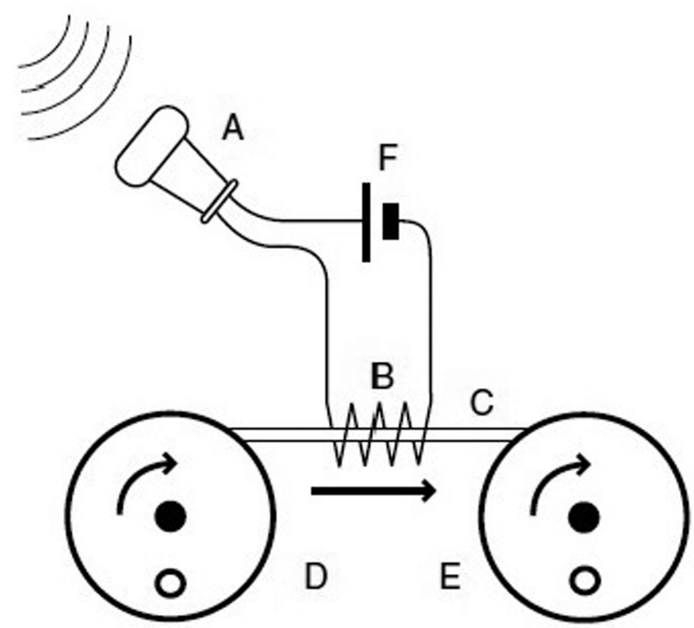

Figura 1: Gravação magnética descrita por Oberlin Smith: A é um microfone, B a cabeça de gravação, C é um fio de aço ou outro meio de gravação, D e E são os carreteis de transporte e F uma bateria 1 .

Pela natureza dos materiais ferromagnéticos, espera-se que a configuração magnética, ou seja, a magnetização de cada segmento de fio (ou cada partícula magnética da fita) permaneça estável desde que a media não seja submetida a campos magnéticos ou aquecida. A informação fica então registrada no suporte de gravação, mais especificamente, no seu estado magnético. Ao aplicarmos um campo magnético a um segmento do fio, a magnetização deste segmento tende a uma orientação de acordo com o campo. Após remoção deste campo, o estado magnético do sistema muda, mas ainda é possível observar uma magnetização tipica não nula na direção do campo aplicado anteriormente. É nesta configuração de magnetização remanescente que a informação fica armazenada.

Depois do processo de gravação, o suporte fica com um padrão de magnetização que deve ser proporcional, em magnitude e direção, ao sinal sonoro original. Se o meio gravado passar com a mesma velocidade $v$ por uma cabeça de reprodução, o fluxo magnético do suporte em movimento irá induzir uma tensão proporcional ao número de voltas $N$ do enrolamento do toróide e a variação de fluxo

$$
V=N \frac{d \phi}{d t}=B_{m} v \operatorname{sen}(\pi g / \lambda)
$$

em que $d \phi / d t$ é a taxa de variação do fluxo magnético, $B_{m}$ é a densidade de fluxo máxima do suporte de gravação, $v$ é a velocidade com que a media passa pela cabeça de reprodução, $g$ é a largura do gap e $\lambda$ é o comprimento de onda do sinal gravado no suporte.

As seções a seguir obedecem uma sequência lógica de acordo com as etapas de registro e leitura. Portanto, primeiro serão apresentadas algumas características de uma cabeça indutora e, em seguida, as características de um suporte de gravação magnética e, por fim, a aplicação 
de uma polarização de corrente adequada na cabeça indutora para melhorar a gravação na media.

\subsubsection{Cabeça indutora}

O principal componente de um gravador magnético é a cabeça indutora de gravação. Assim como um microfone, a cabeça de gravação também é um transdutor eletroacústico, mas de classe II [7]. Considere um núcleo toroidal, com seção transversal circular de raio $r$ e com um gap estreito ( $g a p$ semi-infinito), com enrolamento de $N$ espiras onde circula uma corrente constante $I$, que gera um campo magnético nos polos do gap. A distribuição do potencial escalar $\Phi(x, y)$, ao longo da superfície da cabeça $(y=0)$, pode ser descrita como

$$
\Phi(x, 0)= \begin{cases}-N I / 2, & \text { para } x<-g / 2 \\ x \frac{N I / 2}{g / 2}, & \text { para }-g / 2<x<g / 2 \\ +N I / 2, & \text { para } x>g / 2 .\end{cases}
$$

O campo vetorial $\vec{H}$ sobre a superfície do polo da cabeça pode ser obtido a partir do potencial escalar

$$
\vec{H}=-\nabla \Phi .
$$

O campo magnético de cabeças indutoras é dado pela aproximação de Karlqvist [8]. Os componentes horizontal e vertical do campo magnético de uma cabeça indutora são respectivamente determinados por

$$
H_{x}(x, y)=-\frac{H_{g}}{\pi}\left[\tan ^{-1}\left(\frac{\frac{g}{2}+x}{y}\right)+\tan ^{-1}\left(\frac{\frac{g}{2}-x}{y}\right)\right]
$$

$$
H_{y}(x, y)=\frac{H_{g}}{2 \pi} \ln \left[\frac{\left(g / 2+x^{2}\right)^{2}+y^{2}}{\left(g / 2-x^{2}\right)^{2}+y^{2}}\right]
$$

em que $g$ é o tamanho do gap e $H_{g}$ é o campo magnético no gap (entre os polos da cabeça) 8]. Para cabeças com gap muito estreito a aproximação de Karlqvist reduz a

$$
\begin{gathered}
H_{x}(x, y)=-\frac{H_{g}}{\pi} \frac{y g}{\left(x^{2}+y^{2}\right)} \\
H_{y}(x, y)=\frac{H_{g}}{\pi} \frac{x g}{\left(x^{2}+y^{2}\right)}
\end{gathered}
$$

As cabeças de gravação e reprodução são construídas tradicionalmente de ferro doce, permalloy, ferrite ou sendust. A leitura de cartões magnéticos e discos rígidos, utiliza um princípio análogo ao das cabeças indutoras para gravar e ler informações.

A Figura 2 ilustra o processo de registro. A Figura 3 apresenta o componente horizontal do campo de registro da cabeça indutora para a aproximação de Karlqvist e para uma cabeça com gap muito estreito e a Figura 4 apresenta o componente horizontal do campo da cabeça indutora.

\section{Registro}

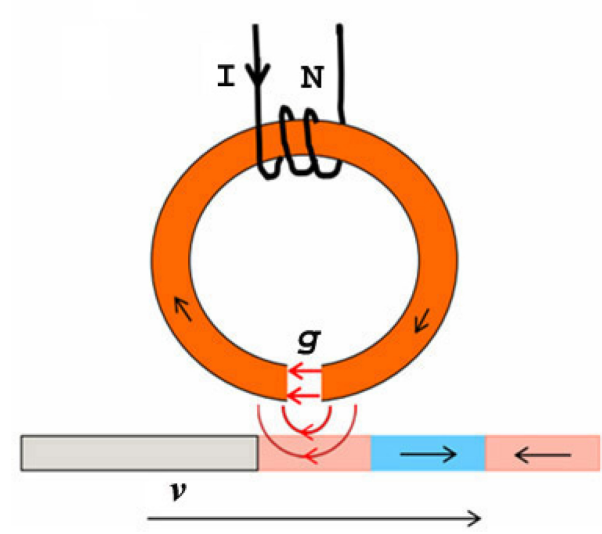

Figura 2: Cabeça indutora de gravação magnética.

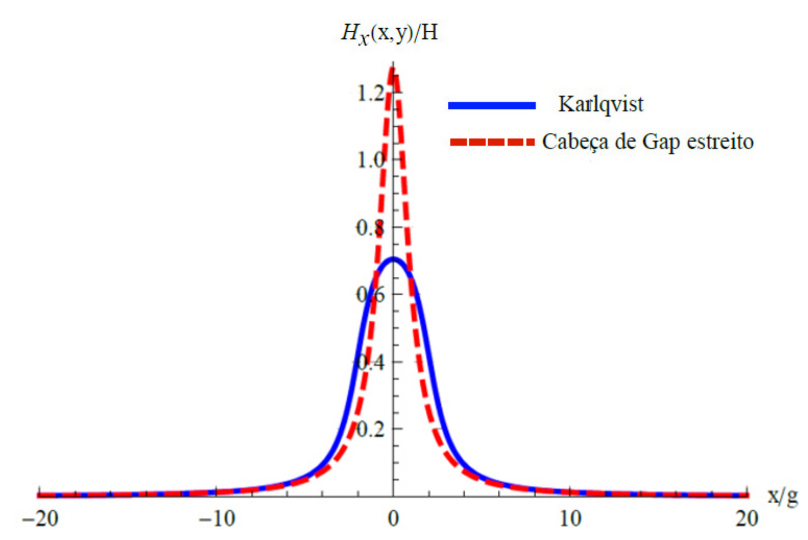

Figura 3: Aproximação de Karlqvist para o componente horizontal do campo da cabeça indutora.

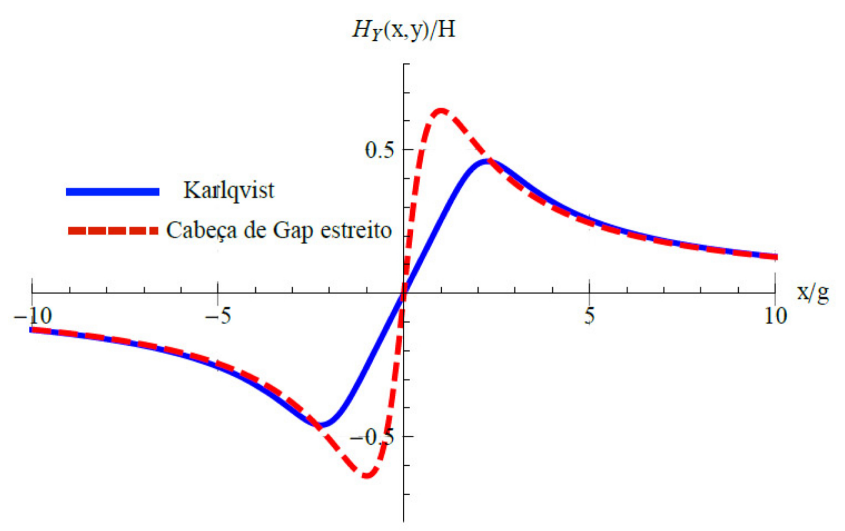

Figura 4: Aproximação de Karlqvist para o componente vertical do campo da cabeça indutora.

\subsubsection{Suporte de gravação (media)}

Ao imergir um material qualquer em uma região onde há um campo magnético $\vec{H}$, a estrutura deste material responde ao campo no qual foi imerso mediante a produção de um campo magnético próprio $\vec{B}$, 


$$
\vec{B}=\mu_{0}(\vec{H}+\vec{M}) .
$$

Nestas condições, dizemos que o material foi magnetizado. A magnetização $\vec{M}$, é a densidade volumétrica dos momentos de dipólo magnético associados à estrutura eletrônica do material. Sua intensidade e orientação dependem do campo externo aplicado e das propriedades do material. Nos materiais magnéticos lineares, a magnetização é proporcional à intensidade do campo magnético, e pode ser escrita em função da susceptibilidade magnética do meio como

$$
\vec{M}=\chi_{m} \vec{H} .
$$

A susceptibilidade magnética $\chi_{m}$ indica a capacidade que um material tem de ser magnetizado quando submetido a um campo magnético externo e é escrita como

$$
\chi_{m}=\left(\mu_{r}-1\right),
$$

em que $\mu_{r}$ é a permeabilidade magnética relativa do material em relação a permeabilidade do vácuo $\mu_{0}, \mu_{r}=$ $\mu / \mu_{0}$. A permeabilidade magnética é uma medida do campo magnético no interior de um material, devido ao campo magnético externo. A classificação magnética dos materiais é feita de acordo com a permeabilidade magnética.

Os suportes magnéticos de gravação, classificados como materiais ferromagnéticos, possuem permeabilidade relativa muito maior que 1 e são fortemente atraídos por campos magnéticos em geral. Os suportes magnéticos de gravação podem sofrer magnetização longitudinal ou vertical [8]. Os componentes do campo magnético gerado por medias magnetizadas longitudinalmente são, respectivamente, dados por

$$
H_{x}^{x}(x, y)=-\frac{M}{\pi}\left[\tan ^{-1} \frac{y+\delta / 2}{x-x_{0}}-\tan ^{-1} \frac{y-\delta / 2}{x-x_{0}}\right],
$$

$$
H_{y}^{x}(x, y)=\frac{M}{2 \pi} \ln \frac{(y+\delta / 2)^{2}+\left(x-x_{0}\right)^{2}}{(y-\delta / 2)^{2}+\left(x-x_{0}\right)^{2}}
$$

em que $M$ é a intensidade da magnetização e $\delta$ é a espessura da media [8].

A Figura 5 ilustra o processo de leitura. A Figura 6 apresenta os componentes $H_{x}^{x}$ e $H_{y}^{x}=0$ ao longo da linha central de uma media magnetizada e a Figura 7 apresenta os componentes $H_{x}^{x}$ e $H_{y}^{x}$ ao longo da superfície da media.

Uma grande dificuldade com os primeiros gravadores magnéticos, foi um nível de distorção que afetava a clareza do som durante a reprodução. Isso ocorre por causa da não linearidade da curva de transferência do suporte de gravação, que corresponde ao fluxo residual resultante depois que o campo externo é removido.

\section{Leitura}

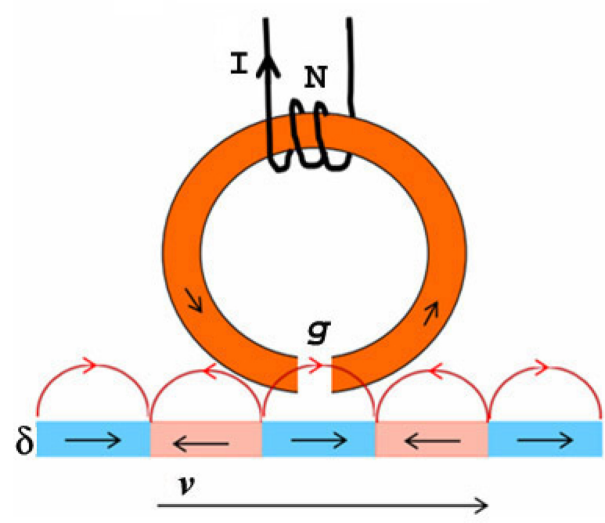

Figura 5: Cabeça de leitura, e suporte com magnetização longitudinal de espessura $\delta$.

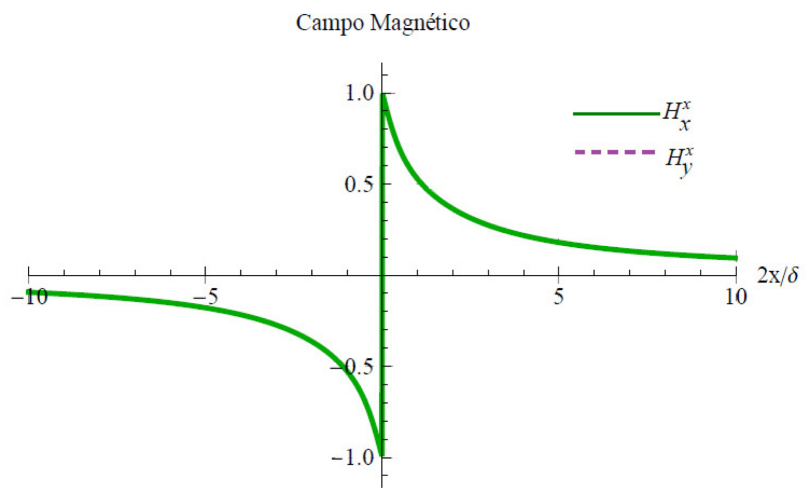

Figura 6: Campo magnético ao longo da linha central da media.

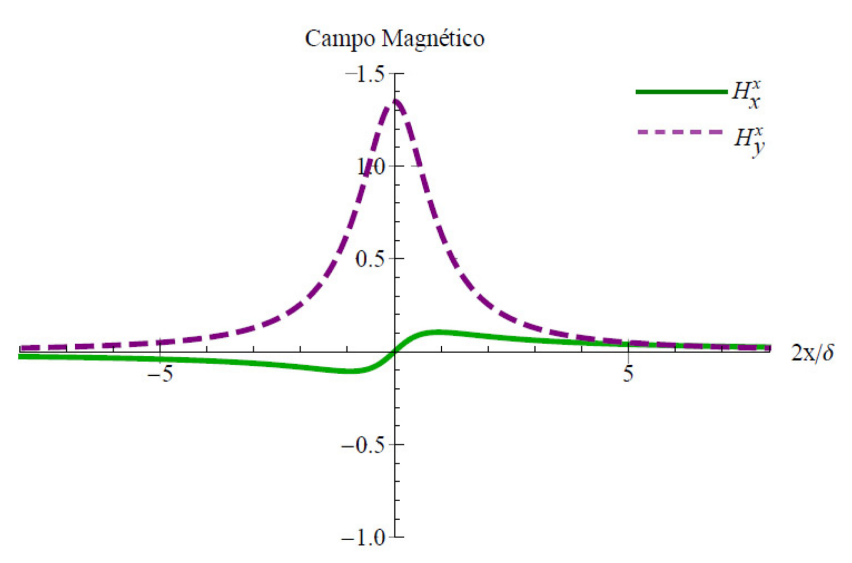

Figura 7: Componentes do campo magnético ao longo da superfície da media.

\subsubsection{Polarização de corrente}

Em 1905, Poulsen e Pederson introduziram um esquema simples de polarização de corrente contínua (polarização CC, ou DC bias) para obter uma gravação melhor. Porém, o ruído da distorção continuava sendo um problema [5].

A solução veio com a polarização por corrente alternada de alta frequência (polarização CA ou $A C$ bias), 
descoberta por Carlson e Carpenter nos EUA em 1921, e novamente por Nagai, Sasaki e Endo no Japão em 1938. Entretanto, a utilização prática na gravação magnética foi introduzida somente a partir de 1940 com a redescoberta de Braunmuehl e Weber na Alemanha. O esquema básico é gerar um sinal CA, em geral, com uma frequência 5 vezes maior que a frequência máxima a ser gravada (por exemplo, $100 \mathrm{kHz}$ para a faixa de áudio de $20 \mathrm{kHz}$ ). O sinal de áudio é então simplesmente adicionado a um sinal de polarização CA 9 .

Para compreender os métodos de polarização $\mathrm{CC}$ e CA, é necessário entender também o que é a curva de transferência. A curva de transferência, no caso da gravação magnética, é o comportamento típico de uma media quando submetida a um campo magnético externo, em que o eixo das abcissas representa a intensidade do campo magnético aplicado $H$, enquanto o eixo das ordenadas indica magnetização residual $M_{r}$ que permanece no sistema após o campo ser removido.

A curva de transferência é obtida através de um processo de magnetização ideal. Considere uma barra de ímã flexível, feito ao cortar um pequeno pedaço da media virgem. Este pedaço pode ser magnetizado por um solenóide com uma corrente contínua conhecida e, após desligar a corrente, o fluxo magnético residual no material, proporcional à magnetização residual, pode ser medido. Ao medir o fluxo residual do material após a remoção da aplicação de diferentes intensidades de campo magnético, obtemos a magnetização residual resultante, que é a curva de transferência da media.

É interessante notar a relação não-linear entre $M_{r}$ e $H$, que é mais acentuada nas regiões de baixos e altos campos magnéticos. Esta não linearidade dificulta o processo de gravacão, pois distorce o sinal original. Portanto, é indispensável evitar estas regiões de campo. No método de polarização CC, o sinal de entrada simplesmente é deslocado para a parte linear da curva, conforme ilustra a Figura 8 .

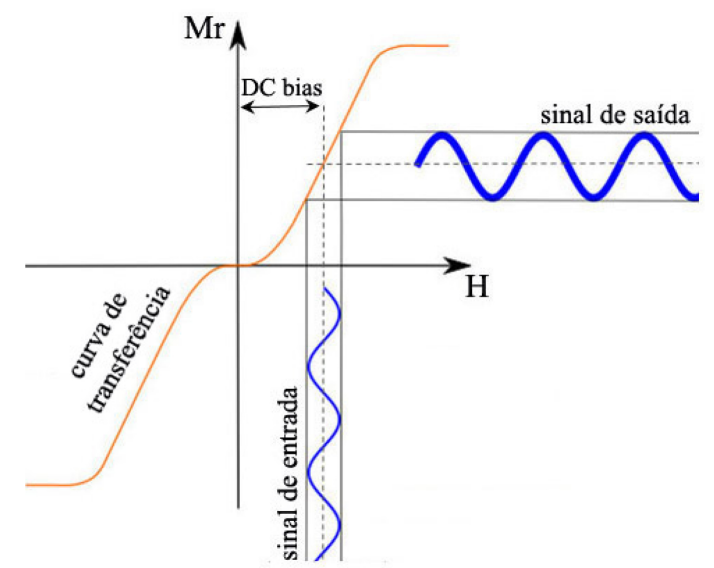

Figura 8: Aplicação de uma polarização de corrente CC para deslocar o sinal de entrada para parte linear da curva de transferência da media.
No método de polarização CA, utiliza-se as duas partes lineares da curva de transferência. A escolha da melhor polarização de corrente CA para a operação prática de um gravador depende de vários fatores, principalmente das dimensões relativas da media, da largura do gap da cabeça indutora e do comprimento de onda registrado [10].

\section{Materiais e métodos}

\subsection{Fios de aço para testes}

Foi utilizado um pedaço de apenas $2 \mathrm{~mm}$ para obter a curva de transferência. Um rolo típico de fio de aço tem aproximadamente $0,15 \mathrm{~mm}$ de diâmetro e $2 \mathrm{~km}$ de comprimento para uma hora de gravação.

\subsection{Recuperação de um gravador de fios}

Para construir a plataforma de gravação magnética de fios, foi utilizado o sistema original (motor e carreteis de transporte) e a cabeça indutora de um gravador WebsterChicago. O gravador de fios foi adaptado para ser utilizado com um pré-amplificador (Presonus, Audiobox 1616VSL) e um computador com software de gravação e edição.

\subsection{Ajuste da polarização de corrente}

Foi utilizado como sinal de entrada um áudio de amplitude constante com variação de $20 \mathrm{kHz}$ a $20 \mathrm{~Hz}$, à uma taxa de uma oitava por 5 segundos. Em seguida, para corrigir a distorção natural da gravação, foi aplicada uma polarização de corrente na cabeça indutora. Primeiro foi adicionado à gravação do sinal de entrada, um sinal de 9V CC com uma fonte (Instruterm, FA-3005). Posteriormente, foi adicionado um sinal de 9V CA de alta frequência com um gerador de funções (Stanford Research, DS345).

A polarização CA deve ser ajustada, começando com uma saída baixa, e aumentar até que o sinal máximo de saída seja observado no osciloscópio ( Tektronix, MSO2024B). Ao continuar a aumentar magnitude da polarização CA até que a saída comece a diminuir (condição de overbias), deve-se retornar a configuração de polarização para saída máxima. O seguimento gravado deve ser reproduzido e monitorado para encontrar a polarização adequada.

\section{Resultados}

\subsection{Caracterização do fio de aço}

A curva de transferência do fio de aço (Figura 9 foi obtida com o Magnetômetro de Gradiente de Campo Alternado (AGFM) do Laboratório de Magnetismo e Materiais Magnéticos (LMMM) da UFSM. O AGFM é um dispositivo capaz de medir variações no momento magnético com alta sensibilidade. Seu princípio de funcionamento baseia-se na força sofrida por um momento 


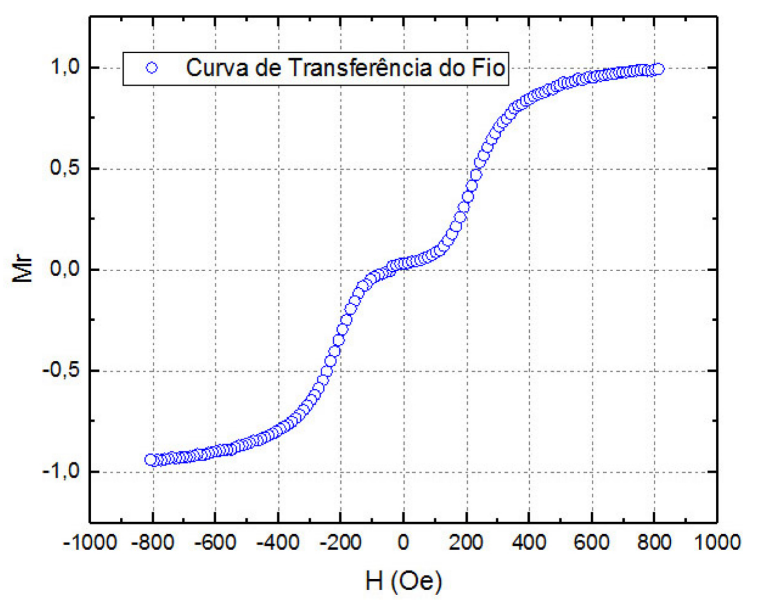

Figura 9: Curva de transferência do fio de aço.

magnético quando submetido a um campo magnético que varia espacialmente. Ao aplicarmos um gradiente de campo magnético alternado, fazemos com que uma amostra magnética presa em uma das extremidades de uma haste vibre. Na outra extremidade existe um material piezoelétrico, que transforma a vibração mecânica em um sinal elétrico. Tal sinal será proporcional à magnetização da amostra 11.

\subsection{Gravador de fios}

A Figura 10 apresenta a foto da plataforma de gravação construída, um gravador de fios Webster-Chicago recuperado, com uma cabeça indutora, carreteis de transporte do fio e uma fonte de energia. O enrolamento do fio é realizado com o movimento vertical da cabeça para cima e para baixo em seu eixo por meio de uma câmara no sistema de acionamento. O movimento da cabeça serve para distribuir o fio uniformemente no carretel de recolhimento.

O sinal de entrada é gravado diretamente na cabeça. A cabeça indutora deve receber sinais de no mínimo $8 \mathrm{~V}$ para começar a gravar. O sinal de entrada sai de

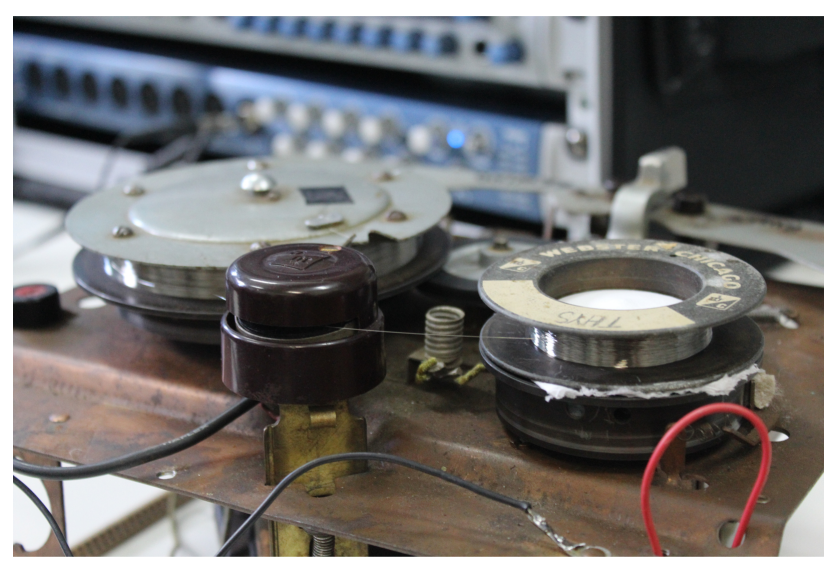

Figura 10: Foto do gravador de fios recuperado. um computador por um cabo P2/P10, passa por um pré-amplificador e é gravado diretamente no fio pela cabeça indutora. A mesma cabeça faz a leitura por um processo recíproco. Em seguida o som gravado no fio, captado diretamente da cabeça de leitura, é enviado para um pré-amplificador conectado a um PC para recuperar digitalmente a gravação analógica realizada no fio. A interface de áudio USB usa conversores de 24 bits e taxa de amostragem de até $96 \mathrm{kHz}$ juntamente com uma estação de trabalho de áudio digital (DAW), um sequenciador que tem a finalidade de gravar, editar e tocar áudio digital, para transferir o som do fio para o fomato .wav.

\subsection{Correção da distorção e relação sinal ruído}

As características das gravações sem polarização e com polarização CC e CA em fios de aço, foram obtidos a partir dos áudios digitalizados. A Figura 11 apresenta os gráficos do sinal de entrada (azul) e dos sinais de saída da gravação sem corrente de polarização (roxo), com polarização CC (laranja) e com polarização CA (vermelho), todos sobrepostos. A Figura 12 apresenta a resposta em frequência do sinal de entrada e dos respectivos sinais de saída. O sinal de entrada e os sinais de saída podem ser escutados em: http://magneticrecording.bandcamp.com

\section{Discussão}

Só foi possível obter melhorias na gravação magnética com a utilização da polarização alternada de alta frequência, que popularizou-se na década de 1940. O principal objetivo da polarização CA, é compensar as nãolinearidades da media e permitir gravações livres de distorções. O ajuste adequado da corrente de polarização também garante uma melhor relação sinal-ruído e uma resposta de frequência ideal para garantir boa fidelidade de gravação. O objetivo, portanto, é obter uma gravação magnética com um sinal forte de saída, com melhora significativa na relação sinal/ruído.

Em baixas frequências, onde o comprimento de onda registrado é longo comparado com o comprimento do gap da cabeça de reprodução e com a espessura do fio, pode-se deduzir que o fluxo gravado é reproduzido sem erro.

As medições de distorção foram realizadas a uma frequência de $250 \mathrm{~Hz}$. Devido à simetria, ocorrem apenas harmônicos ímpares, em que o terceiro harmônico é predominante. A razão entre as amplitudes do terceiro harmônico e a fundamental no sinal de saída pode ser utilizada como uma medida da distorção. Para os sinais de saída, verifica-se que, se a distorção harmônica não exceder $2 \%$, dificilmente será perceptível.

Ao obter medidas de um sinal em meio ruidoso, a relação sinal/ruído (RSR) é definida como a razão da potência de um sinal e a potência do ruído sobreposto ao sinal. A relação sinal/ruído obtida foi de $33,75 \mathrm{~dB}$ 


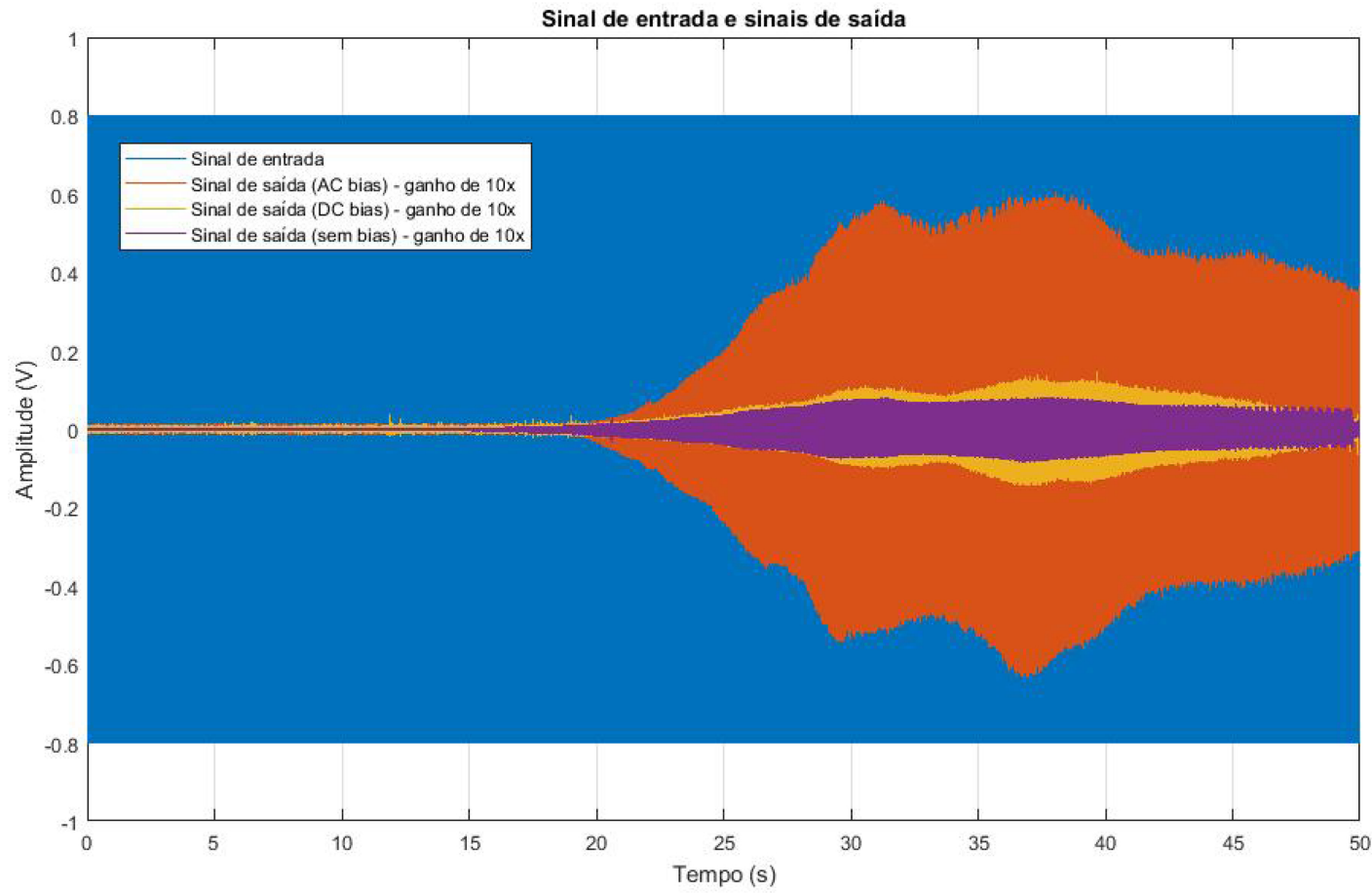

Figura 11: Sinal de entrada e sinais de saída sobrepostos.

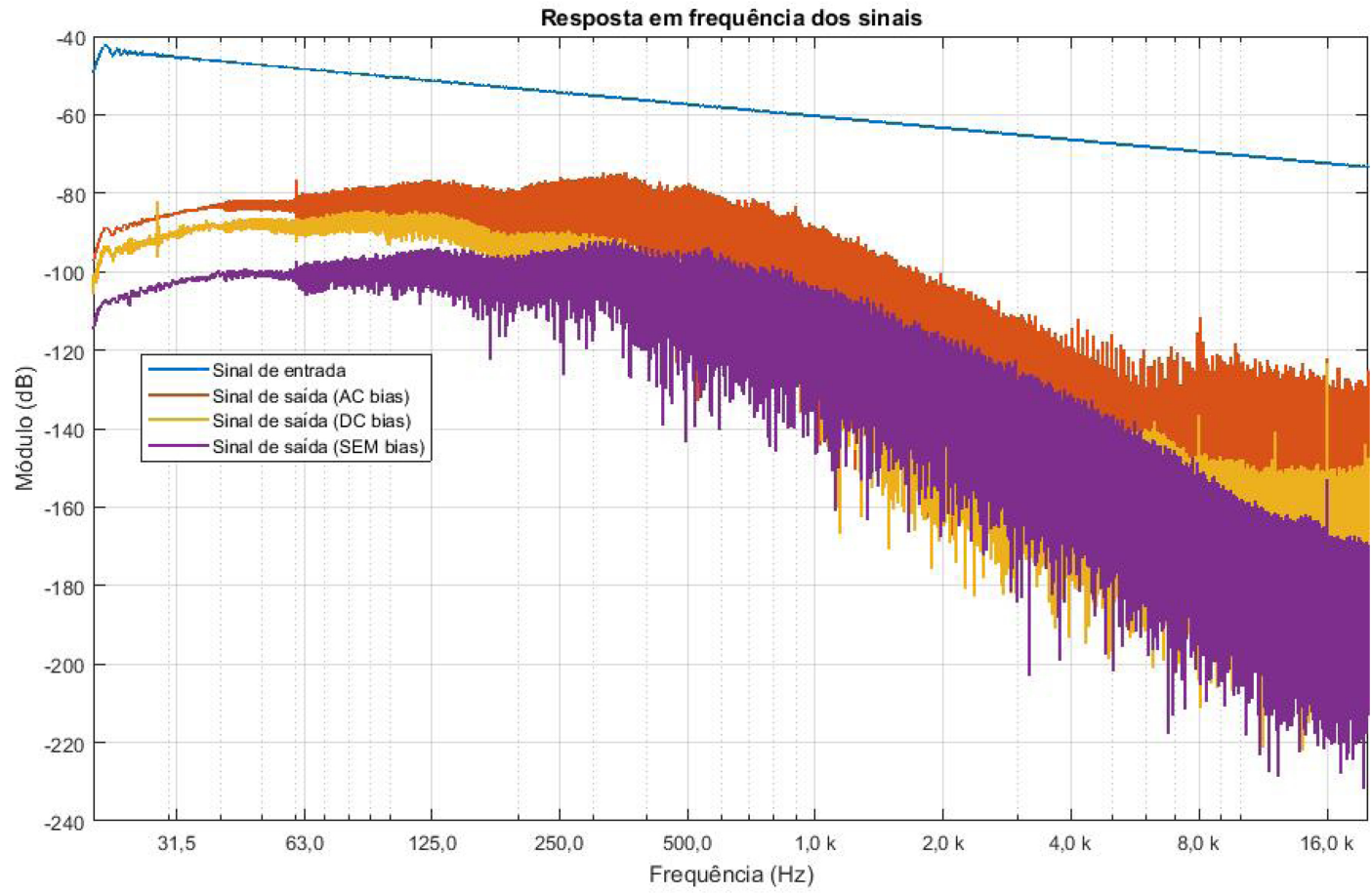

Figura 12: Resposta em frequência do sinal de entrada e dos sinais de saída.

com gravação magnética sem polarização de corrente. Com a polarização CC, uma vez que apenas uma porção pequena da curva de magnetização é utilizada, o sinal de saída é fraco em relação ao ruído natural do suporte de gravação, ou seja, temos uma relação sinal/ruído fraca, de 20,38 dB. Com a polarização CA, a relação sinal ruído obtida foi de 33,13 dB e verificou-se que uma condição de polarização baixa ou alta, direciona o sinal para as regiões não lineares da curva de transferência do fio e causa distorção.

A relação sinal-ruído geralmente é adotada para indicar a faixa dinâmica de um equipamento, ou seja, a gama de intensidades que podem ocorrer, e que vai desde o menor sinal (na escala próxima do ruído) até o má- 
ximo sinal sem distorção. Além da distorção e da fraca relação sinal/ruído do fio de aço, a primeira media magnética inevitavelmente foi substituída por outra com maior capacidade de armazenamento, a fita magnética.

\section{6. conclusão}

Revisamos os princípios dos sistemas de registro de som por gravação magnética a partir da reconstrução de um gravador de fios. O áudio foi captado diretamente da cabeça de leitura através de um pré-amplificador conectado a um computador. Comparamos o sinal gravado direto da cabeça de gravação com polarização CC e polarização CA para fios de aço. A distorção harmônica total e a relação sinal/ruído foram determinadas e discutidas para cada situação. Foi possível verificar experimentalmente a condição ideal de configuração de polarização de corrente alternada de alta frequência para obter uma gravação com maior fidelidade possível.

\section{Agradecimentos}

Aos alunos da disciplina EAC1028 - Técnicas de Gravação, do curso de Engenharia Acústica, do Centro de Tecnologia da Universidade Federal de Santa Maria.

\section{Referências}

[1] N.E. Souza Filho, J. Rosa, D.M. Silva e N.G.C. Astrath, Revista Brasileira de Ensino de Física 44, e3601 (2018).

[2] O. Smith, Electrical World 2, 116 (1888).

[3] V. Poulsen, US Patent 661,619 (1900).

[4] M. Clark e H. Nielsen, Business History Review 69, 41 (1995)

[5] E.D. Danioel, C.D. Mee e M.H. Clark, Magnetic recording: the first 100 years (John Wiley \& Sons, Hoboken, 1999).

[6] J.M.D. Coey, Magnetism and Magnetic Materials (Cambridge University Press, Cambridge, 2010).

[7] A.F. Fischer e N.E. Souza Filho, Fundamentos de Eletroacústica (Editora Scienza, São Carlos, 2018).

[8] H.N. Bertram, Theory of magnetic recording (Cambridge University Press, Cambridge, 1994).

[9] J.G. McKnight, Electronics World 78, 34 (1967).

[10] W.K. Westmijze, Philips Research Reports 8, 245 (1953).

[11] P.J. Flanders, Journal of Applied Physics, 63, 3940, (1988). 